\section{UNINTENTIONAL CHILDHOOD INJURIES IN KENYA: EPIDEMIOLOGICAL PATTERNS BASED ON HOSPITAL TRAUMA REGISTRY DATA}

${ }^{1}$ Huan He, ${ }^{1}$ Yuen W Hung, ${ }^{1}$ Isaac M. Botchey, ${ }^{1}$ Abdul M Bachani, ${ }^{2}$ Hassan Saidi, ${ }^{1}$ Adnan A Hyder, ${ }^{1,3}$ Kent A Stevens. 'Johns Hopkins International Injury Research Unit, Johns Hopkins Bloomberg School of Public Health, USA; ${ }^{2}$ University of Nairobi, Kenya; ${ }^{3}$ Johns Hopkins Hospital Department of Surgery, USA

\subsection{6/injuryprev-2016-042156.927}

Background Unintentional injuries are the leading cause of death for children under age 18 years globally. Our study aims to describe the epidemiological patterns of unintentional injuries and assess the adoption of safety measures and pre-hospital care among paediatric injury patients to inform future interventions in Kenya.

Methods The Trauma Registry System collected data of injury patients from four major hospitals in Kenya between January 2014 and July 2015. Out of the 4257 paediatric patients, 4005 (94.1\%) with unintentional injuries were included in analysis.

Results We found that boys were more likely to suffer from unintentional injuries than girls at all ages, accounting for $60.4 \%$ of those under 5 years $(\mathrm{n}=1359), 68.0 \%$ of those aged 5-9 years $(\mathrm{n}=1130)$, and $73.0 \%$ of those aged $10-17$ years $(n=1516)$. Majority of the injuries happened at home $(79.9 \%)$ or on the road $(14.5 \%)$ for those under age 5 ; while more injuries occurred at school for those aged 5-9 (15.0\%) and aged 10$17(31.3 \%)$. The top three injuries were fall (46.5\%), burn (29.1\%), and road traffic injuries $(14.2 \%)$ for children under age 5 years, and fall (64.8\%), traffic injuries (19.1\%), and struck or hit by a person or object (7.3\%) for children aged 5-17 years. Death rate was higher for the under age 5 group (4.9\%) than that for the age 5-17 group (1.3\%). Burns and traffic injuries had higher death rates $(6.6 \%, 5.6 \%)$ and severity scores compared to the other injuries. The adoption rates for helmets and seatbelts were about $10 \%$ in at risk patients. Only $18.4 \%$ children received pre-hospital care, which was mostly provided by relatives and friends and associated with lower odds of death $(p<0.05)$.

Conclusions Our preliminary findings show that the epidemiological patterns of injuries differ by child age, which implicates that interventions should be tailored for specific age phases. This study pinpoints the needs to prioritise promoting pro-safety behaviours to alleviate the consequences of injuries in Kenya.

\section{DEVELOPMENT AND VALIDATION OF A SURVEY ON FATHERS' ATTITUDES TOWARDS CHILD INJURY PREVENTION}

Lise Olsen, Mariana Brussoni, Takuro Ishikawa, Louise Masse, Grace Chan. University of British Columbia, Canada

\subsection{6/injuryprev-2016-042156.928}

Background The purpose of this study was to develop and validate a survey instrument to measure fathers' attitudes regarding injury prevention for their children. Parents play an important role in reducing child injury risk; however, our understanding of the unique role that fathers play in keeping children safe is limited. Our previous research indicated that fathers believed risk played an important role in their children's development and that they aimed to balance these risks with protecting children from injury.

Methods We developed a survey to measure two main dimensions of fathers' parenting attitudes: child protection from injury
(16 items) and child risk engagement (24 items). The survey content was created using a 4-step process utilising expert feedback, cognitive interviews with fathers and pilot-testing. The survey was administered to fathers of children ages 6-12 years visiting the Emergency Department of a children's hospital in British Columbia, Canada. We used confirmatory factor analysis to validate the factor structure of the questionnaire.

Results There were 302 fathers who completed the questionnaire, with $45 \%$ children attending hospital for an injury and $55 \%$ for another medical concern. Following analysis, the child protection from injury factor was found to consist of eight items (Cronbach's alpha of 0.75 ) and the child risk engagement factor consisted of six items (Cronbach's alpha of 0.77). Correlation between the factors was low suggesting the dimensions are independent.

Conclusions These survey data provide support for the idea that for fathers, child protection from injury and child risk engagement reflect distinct attitudinal dimensions. These results add to our understanding of fathers' conceptions related to child safety and offer direction for development of injury prevention messages and interventions that are tailored to fathers' perspectives and experiences.

\section{MULTI-PROFESSIONAL COLLABORATION IN THE SERVICE OF CHILDREN AND YOUNG PEOPLE}

1,2Tuija Leppäkoski, 1,2Eija Paavilainen, ${ }^{3}$ Sami Perälä, ${ }^{3}$ Minna Koivuluoma, 2,4 Johanna SorvettulaTiina Pekkanen. 'University of Tampere, Finland; ${ }^{2}$ South Ostrobothnia Hospital District, Finland; ${ }^{3}$ South Ostrobothnia Healthtechnology Development Centre, Finland; ${ }^{4}$ City of Seinäjoki, Finland

\subsection{6/injuryprev-2016-042156.929}

Background Families' various problems are reflected in the everyday life of children and young people. In order to help them, low-threshold services based on multi-professional collaboration between the various authorities are required. Within the framework of the 'Code of caring' project (www.välittämisenkoodi.fi), a new operational model and communications system will be developed, providing responses to children's and young people's challenging life situations, preventive marginalisation and violence and promoting the safety of children and young people.

Methods The impact of the new operational model on aid provision to children and young people aged $12-17$ will be studied with the help of a three-phase research process. The first phase (2014) will comprise the gathering of national data on the marginalisation of children and young people. The second phase (2014-2015) will study the current situation as assessed by the various authorities themselves and as experienced both by the children and young people. The third phase (2014-2015) will describe the views and experiences expressed by the various authorities and children and young people of working under the new model. The experiences of children and young people will be studied with the help of thematic interviews as well as queries sent to the actors involved. The data thus gathered will be analysed using both qualitative and quantitative methods.

Results The results obtained from the first survey indicate that the representatives of the authorities were almost unanimous in believing that common conversations aid in understanding the overall process aimed at helping children and young people. Themes represented in development suggestions included the need for common training, in order to better understand the roles of the different helpers and the goals set for their work. 